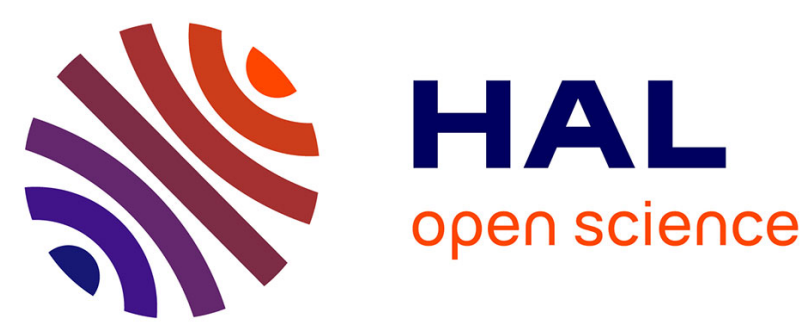

\title{
Sentiment Analysis of Movie Reviews Based on CNN-BLSTM
}

Qianzi Shen, Zijian Wang, Yaoru Sun

\section{To cite this version:}

Qianzi Shen, Zijian Wang, Yaoru Sun. Sentiment Analysis of Movie Reviews Based on CNN-BLSTM. 2nd International Conference on Intelligence Science (ICIS), Oct 2017, Shanghai, China. pp.164-171, 10.1007/978-3-319-68121-4_17. hal-01820937

\section{HAL Id: hal-01820937 https://hal.inria.fr/hal-01820937}

Submitted on 22 Jun 2018

HAL is a multi-disciplinary open access archive for the deposit and dissemination of scientific research documents, whether they are published or not. The documents may come from teaching and research institutions in France or abroad, or from public or private research centers.
L'archive ouverte pluridisciplinaire HAL, est destinée au dépôt et à la diffusion de documents scientifiques de niveau recherche, publiés ou non, émanant des établissements d'enseignement et de recherche français ou étrangers, des laboratoires publics ou privés. 


\title{
Sentiment Analysis of Movie Reviews Based on CNN- BLSTM
}

\author{
Qianzi Shen ${ }^{1}$, Zijian Wang ${ }^{1}$, Yaoru Sun ${ }^{1, *}$ \\ ${ }^{1}$ Department of Computer Science, \\ Tongji University, Shanghai, China \\ sqzetongji.edu.cn, $1410482 @$ tongji.edu.cn, yaorultongji.edu.cn*
}

\begin{abstract}
Sentiment analysis has been a hot area in the research field of language understanding, but complex deep neural network used in it is still lacked. In this study, we combine convolutional neural networks (CNNs) and BLSTM (bidirectional Long Short-Term Memory) as a complex model to analyze the sentiment orientation of text. First, we design an appropriate structure to combine CNN and BLSTM to find out the most optimal one layer, and then conduct six experiments, including single CNN and single LSTM, for the test and accuracy comparison. Specially, we pre-process the data to transform the words into word vectors to improve the accuracy of the classification result. The classification accuracy of $89.7 \%$ resulted from CNN-BLSTM is much better than single CNN or single LSTM. Moreover, CNN with one convolution layer and one pooling layer also performs better than $\mathrm{CNN}$ with more layers.
\end{abstract}

Keywords: Natural Language Processing, CNN, LSTM, Sentiment Analysis

\section{Introduction}

As the significant carries of emotion, texts are in an irreplaceable position on the Internet. The sentiment analysis is usually used to measure the emotional attitude of texts and related studies grow quickly. In the literature, Hazivassiloglou and McKeown investigated semantic orientation of adjectives based on cluster methods [1]. Turney and Littman chose seven pairs of words that have strong orientation and then set SO-PMI (semantic orientation-pointwise mutual information) to judge a word with a standard word. According to SO-PMI, they chose two groups of words in which one is positive ( $\mathrm{P}$ word ) and the other is negative ( $\mathrm{N}$ word ) as standard words [2]. Kim chose a method based on sentiment knowledge and summed up the weighing of phrases and describing words [3]. Pang studied sentiment analysis of English texts with machine learning [4]. With the development of artificial intelligence and deep learning, more and more related networks and methods have been involved in natural processing. In a variety of networks, CNN [5] is widely used for its generalization. Because of its advantage in learning the higher level features, $\mathrm{CNN}$ has achieved a great breakthrough [6] and being applied to many areas, such as image classification, face recognition and other tasks related to image [7]. Among them, LSTM [8] is a kind of recurrent neural 
networks and is able to keep the information for a long time because it has memory cells. In other words, LSTM is a time-order network [9],while BLSTM (Bidirectional Long Short Term Memory) is a kind of network combining LSTM with BRNN [10], which has two directions to input data and two hiding layers to save the information in both directions and sharing the same output layer. In this paper, we designed a more complex model containing both CNN and BLSTM for sentiment analysis.

The rest of the paper is organized as follows: Section 2 reviews the related work to CNN and LSTM. Section 3 describes the model we used in this work. Section 4 shows the experiments and their results. Section 5 presents the conclusion and future works.

\section{$2 \quad$ Methods and Models}

\section{$2.1 \quad \mathrm{CNN}$}

Usually, convolutional neural networks[5] are used in image classification. In this study, CNN was used as a feature important part in our sentiment analysis model.

Convolutional neural networks provide a learning model which is end to end and the parameters of the model can be trained with gradient descent algorithm. A typical convolutional neural network consists of an input layer, convolution layers, pooling layers, a full connection layer and an output layer. Its architecture is shown in Figure 1.

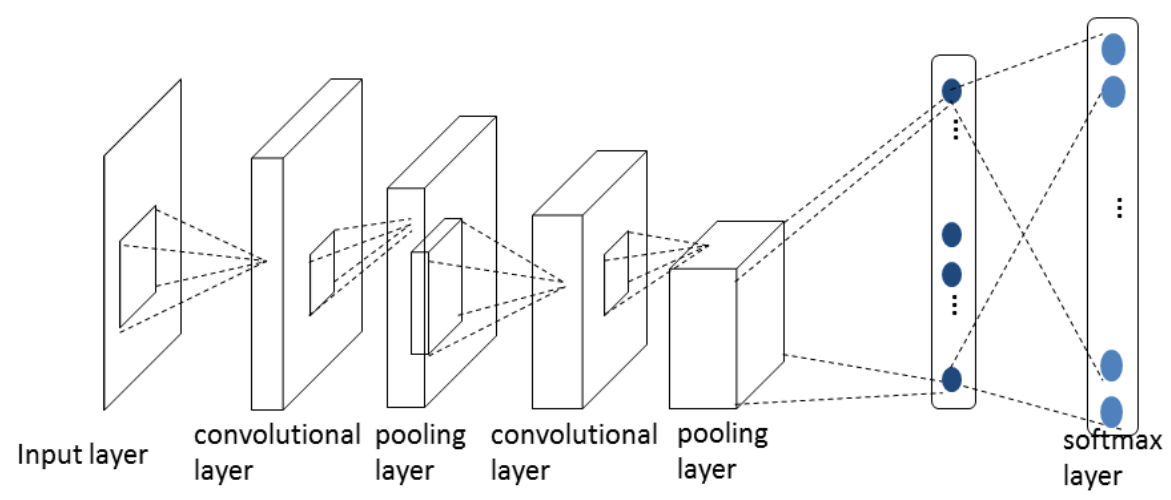

Fig. 1. structure of typical CNN.

Both images and texts can be presented as a matrix. The matrix $\mathrm{V}$ is the input of a $\mathrm{CNN}$. We suppose that $H_{i}$ is the $i$ layer of the network ( $V=H_{0}$ ).

If $H_{i}$ is a convolutional layer:

$$
H_{i}=f\left(H_{i-1} \odot W_{i}+b_{i}\right)
$$

In formula (1) $W_{i}$ is the weight vector of $H_{i}$ layer. Operator $\odot$ is the convolution operation of the convolution kernel and the $H_{i-1}$ layer. The output of the convolution 
will be added with the offset $b_{i}$. And finally, $H_{i}$ could be calculated by a nonlinear excitation $f(x)$.

If $H_{i}$ is a pooling layer:

$$
H_{i}=\operatorname{subsampling}\left(H_{i-1}\right)
$$

The purpose of this layer is to reduce dimensionality and keep the features stable to some extent.

What we want is a probability distribution $Y$. With several convolution layers and pooling layers, we get a model:

$$
Y(i)=P\left(L=l_{i} \mid H_{0} ;(W, b)\right)
$$

Gradient descent algorithm is used to train the parameters $(W$ and $b)$ of each layer.

$$
\begin{aligned}
E(W, b) & =L(W, b)+\frac{\lambda}{2} W^{T} W \\
W_{i} & =W_{i}-\eta \frac{\partial E(W, b)}{\partial W_{i}} \\
b_{i} & =b_{i}-\eta \frac{\partial E(W, b)}{\partial b_{i}}
\end{aligned}
$$

$\lambda$ is the parameter to control the overfitting and $\eta$ is the learning rate.

\subsection{LSTM and BLSTM}

LSTM [8] is a kind of recurrent neural networks (RNN). Because of its ability to make use of long term information, it is suitable for sequential data processing in texts. LSTM unit is shown in Figure 2. And BLSTM [10] is included in LSTM, which is bidirectional.

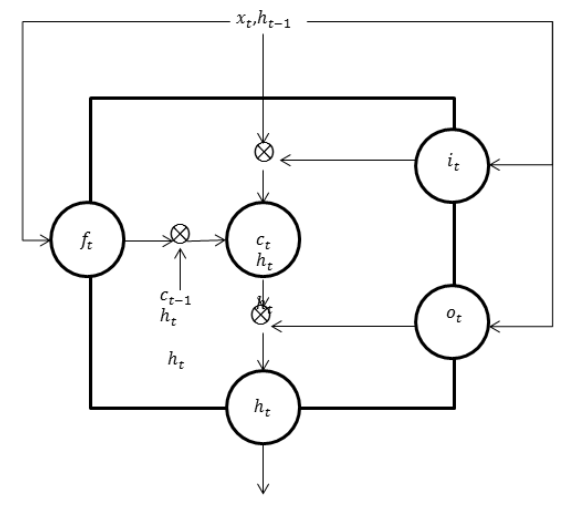

Fig. 2. LSTM memory block.

A LSTM memory block unit contains three gates, input gate, forget gate and output gate. These gates facilitate saving, reading, resetting and updating the long term information. We supposed $\mathrm{x}$ as the input, $\mathrm{c}$ as the memory cell and the $\mathrm{h}$ as the output. We calculate the candidate cell $\tilde{c}_{t}, W_{x c}$ and $W_{h c}$ are weights of input and the output from previous time.

$$
H_{i}=f\left(H_{i-1} \odot W_{i}+b_{i}\right)
$$


Input gate value $i_{t}$ is used to control the impact of memory cell. Forget gate value $f_{t}$ is used to control the impact from history information to current memory. And output gate value is used to control the output of the unit.

$$
\begin{gathered}
i_{t}=\sigma\left(W_{x i} x_{t}+W_{h i} h_{t-1}+W_{c i} c_{t-1}+b_{i}\right) \\
f_{t}=\sigma\left(W_{x f} x_{t}+W_{h f} h_{t-1}+W_{c f} c_{t-1}+b_{f}\right) \\
o_{t}=\sigma\left(W_{x o} x_{t}+W_{h o} h_{t-1}+W_{c o} c_{t-1}+b_{o}\right)
\end{gathered}
$$

$x_{t}$ is the current input value. $h_{t-1}$ is the previous output value. And $c_{t-1}$ is the previous memory cell. $\sigma$ is an activation function that we usually choose logistic sigmoid algorithm for it.

$$
\begin{array}{r}
c_{t}=f_{t} \otimes c_{t-1}+i_{t} \otimes \tilde{c}_{t} \\
h_{t}=o_{t} \otimes \tanh \left(c_{t}\right)
\end{array}
$$

\section{CNN-BLSTM model}

\subsection{Word embedding}

Word embedding is a significant technology in NLP when referring to deep learning. It turns each word into a feature embedding containing semantic information. In this study, word embedding was calculated with GloVe algorithm( Global Vector for word Representation) which is represented by Stanford in 2014[11].

it's the loss function of GloVe:

$$
J(\theta)=\frac{1}{2} \sum_{i, j=1}^{W} f\left(P_{i j}\right)\left(u_{i}^{T} v_{j}-\log P_{i j}\right)^{2}
$$

In this formula, $f(x)$ is a truncation function to reduce the disturbance of frequently used words.

\subsection{CNN-BLSTM model}

In this paper, our model combined one-layer CNN and BLSTM. Each word in the texts has been processed to be a 50-dimensional vector in the preprocessing of the model. The embedding processing is shown in Figure 1.

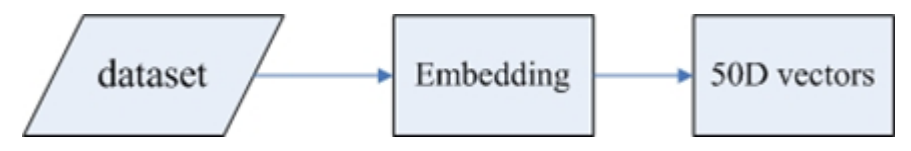

Fig. 3. word embedding.

The pretrained words in the dataset were projected into word vectors in input part of the our model. A convolution layer, a maxpolling layer, a dropout layer and a BLSTM layer was followed sequentially. The whole structure is shown in Figure 2. 


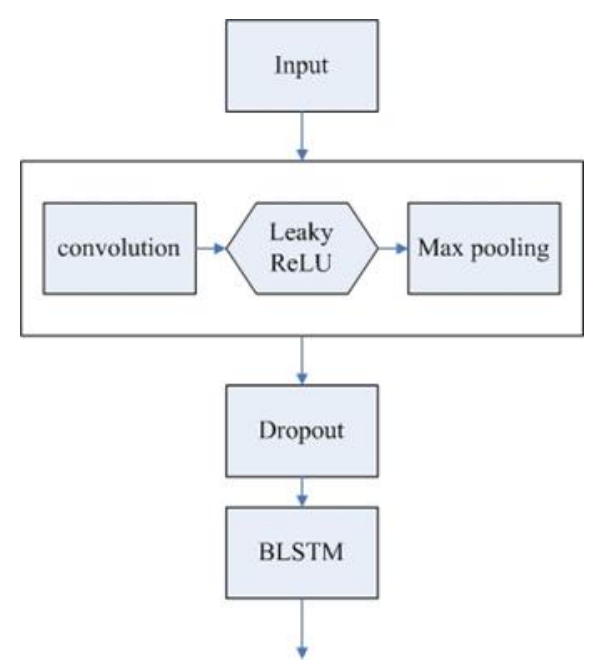

Fig. 4. CNN\&BLSTM model structure.

The input is the result from word embedding process. The input layer receive a maximum of 1000 word vectors, each of them has 50 dimensions. We used convolution kernel size of 5 in CNN layer with activation function of LeakyRelU, a max pooling layer followed it with pool size of 4 . In order to prevent model overfitting, we used a dropout layer after the last max pooling layer. The last part of the model is a BLSTM layer and two dense in which the last dense used softmax as activation and output 2 dimension vector refer to probabilities of the positive and negative classes.

\section{$4 \quad$ Experiments and results}

\subsection{Dataset}

We used the dataset about the sentiment classifications of movie reviews in IMDB. The labeled dataset consists of 50000 IMDB movie reviews. They only have two types of reviews, positive or negative. The whole dataset was divided into two parts. 40000 labeled reviews were training set and the other 10000 were test set.

\subsection{Experiments}

\subsubsection{Experiment 1.}

We compared simple BLSTM and CNN-BLSTM model for sentiment classifications. BLSTM model used a BLSTM layer with 128 unit outputs. CNN-BLSTM model connected a CNN layer with the size of 5-unit kernel, a max pooling layer with pool size 4 and an BLSTM layer with 128-unit output. The maximum feature numbers are 20000, maximum lengths are 1000 and the loss function is categorical cross entropy in the both models. The results are shown in Table 1. 
Table 1. Comparison of BLSTM and CNN-LSTM.

\begin{tabular}{lll}
\hline & BLSTM & CNN-BLSTM \\
\hline Accuracy & $82.5 \%$ & $85.3 \%$ \\
\hline
\end{tabular}

The accuracy of each single model is respectively $82.5 \%$ and $85.3 \%$. CNN-BILSTM performed better than BLSTM model. The local semantic information in a sentence extracted by the convolution layer could lead to the better performance of CNNBLSTM.

\subsubsection{Experiment 2.}

We compared six different models. Three of them are models with respectively 1,2 or $3 \mathrm{CNN}$ layers with a BLSTM layer and a full connected layer with pre-trained word embedding (using GloVe, dimension: 50), and the others are models without pretrained word embedding. In every model, each CNN layer used 64 filters with kernel size 5, each CNN layer was followed by a max-pooling layer with pool size 4, the last pool layer was followed by a dropout layer and a BLSTM layer with 128 unit outputs, the last layers were two full connected layers with respectively 128 unit outputs / activation function of LeakyReLU and 2-unit outputs / activation function of softmax. Every model was trained with 10 epochs, the training batch size is 128 with loss of categorical cross entropy and RMSProp optimizer. The comparison results are shown in Table 2.

Table 2. Comparison of 6 models.

\begin{tabular}{lrrrr}
\hline & 1CNN- & 2CNN- & \multicolumn{1}{l}{ 3CNN- } & Average \\
& BLSTM & BLSTM & \multicolumn{1}{c}{ BLSTM } & \\
\hline With pretrained word embedding & $89.7 \%$ & $86.2 \%$ & $77.7 \%$ & $84.5 \%$ \\
Without pretrained word embed- & $85.3 \%$ & $81.6 \%$ & $79.1 \%$ & $82.0 \%$ \\
ding & & & & \\
Accuracy & $87.9 \%$ & $83.9 \%$ & $78.4 \%$ & \\
\hline
\end{tabular}

The results showed that the 1CNN-BLSTM model with pretrained word embedding got the best accuracy $89.7 \%$, and the 3CNN-BLSTM model with pretrained word embedding got the lowest accuracy $77.7 \%$.

At first we could compare the models with pretrained word embedding and models without those, the result showed that average accuracy of the former is $84.5 \%$ while average accuracy of the latter is $82.0 \%$. It's obviously that the pretrain word embedding enhanced the performance of CNN-BLSTM, which could be caused by the extra semantic information brought by word embedding.

We could also compared models with different numbers of CNN layer. It was kind of weird that the models with more $\mathrm{CNN}$ layer got worse performance. This consequence might be on the contrary of the cognition of us that the model with more complex structure should perform better. But it's known that IMDB datasets only have 50000 samples (40000 for training and 10000 for testing). The consequence could be the results of under-fitting of the complex model. 


\section{$5 \quad$ Conclusion and Future Work}

In this work, we described a series of experiments with CNN and BLSTM and obtained a satisfactory accuracy that could reach up to $89.7 \%$. The whole process contained two steps. The first step was the data pre-processing and each word was turned into a 50D word vector. The result was obviously better if we did word embedding before the data was put into our model directly.

The second step was to accomplish the sentiment analysis with CNN and BLSTM. In this step, we changed the numbers of layers of convolution and pooling in CNN to find a simple CNN with one layer of convolution and one layer of pooling which performed best by comparison. The accuracy of two-layer CNN which contained two convolution layers and two pooling layers was $86.2 \%$ and the accuracy of three-layer CNN which contained three convolution layers and three pooling layers was $77.7 \%$.

In the model, the dropout function was also added to make the result reliable because of its reduction of over fitting. We also investigated whether the additional pre-processing of the data could give the result an advantage like adding POS (part of speech) tagging to the experiment, because not all of the words make sense. In general, adjectives and adverbs describe the feelings of the authors and therefore in the future work, we will devote to exploring more significant methods to do pre-processing of the natural language data.

\section{Acknowledgment}

This work was supported by the Grant from the National Natural Science Foundation of China (61173116).

\section{References}

1. Hatzivassiloglou, V., McKeown, K. R.: Predicting the semantic orientation of adjectives. C. Proceedings of the eighth conference on European chapter of the Association for Computational Linguistics. Association for Computational Linguistics, pp. 174-181 (1997)

2. Turney, P. D.: Thumbs up or down Semantic orientation applied to unsupervised of reviews. Proceedongs of 40th Annual Meeting of the Association for Computation Linguistics. Somerset: ACL, pp. 417-424 (2002).

3. Kim, S. M., Hovy, E.: Automatic detection of opinion bearing words and sentences. C. In Companion Volume to the Proceedings of the International Joint Conference on Natural Language Processing (IJCNLP) Vol.8 (2005).

4. Pang, B., Lee, L., Vaithyanathan, S.: Thumbs up?: sentiment classification using machine learning techniques.. C. pp. 79-86 (2002).

5. Bouvrie, J. Notes on convolutional neural networks. (2006).

6. LeCun, Y., Bengio, Y., Hinton, G.. Deep learning. J. Nature, 521(7553), pp. 436-444 (2015).

7. Gu, J., Wang, Z., Kuen, J., Ma, L., Shahroudy, A., Shuai, B., Wang, G.: Recent advances in convolutional neural networks. J. arXiv preprint arXiv:1512.07108 (2015). 
8. Hochreiter, S., Schmidhuber, J.: Long short-term memory. J. Neural computation, 9(8): pp.1735-1780 (1997).

9. Sundermeyer, M., Ney, H., Schlüter, R.: From feedforward to recurrent LSTM neural networks for language modeling. J. IEEE/ACM Transactions on Audio, Speech and Language Processing (TASLP), 23(3): 517-529 (2015).

10. Schuster, M., Paliwal, K. K.: Bidirectional recurrent neural networks. J. IEEE Transactions on Signal Processing, 45(11): 2673-2681 (1997).

11. Pennington, J., Socher, R., Manning, C. D.: C. Glove: Global Vectors for Word Representation. EMNLP. 14: 1532-1543 (2014). 